Keiichi Ohta, DDS

Department of Dentistry and Oral Surgery, Unit of Sensory and Locomotor Medicine, Division of Medicine, Faculty of Medical

Sciences, University of Fukui, Fukui, Japan
Hitoshi Yoshimura, DDS, PhD

Department of Dentistry and Oral Surgery, Unit of Sensory and Locomotor Medicine, Division of Medicine, Faculty of Medical

Sciences, University of Fukui, Fukui, Japan

\title{
Leukoplakia of the tongue
}

\begin{abstract}
A
75-YEAR-OLD MAN presented to the dentistry and oral surgery department with an asymptomatic white spot on the right lateral border of the tongue that had been present for an unknown period of time. He had a history of hypertension. He drank alcohol almost every day, but he had never smoked.

Intraoral examination showed a homogeneous flat white plaque with slight corrugations measuring $45 \mathrm{~mm}$ by $20 \mathrm{~mm}$ (Figure 1), with no induration or tenderness on palpation. Extraoral examination showed no cervical lymphadenopathy. Blood testing and swab culture from the lesion revealed no significant abnormalities.

Incisional biopsy was performed, and histopathologic study showed squamous epithelial hyperplasia with parakeratosis consistent with leukoplakia. We suggested surgical excision to allow a complete histopathologic study of the lesion, but the patient requested observation only, because of the risks of paresthesia, dysgeusia, and motor dysfunction of the tongue. Three repeat biopsies over the next 14 months showed no significant change in the lesion.
\end{abstract}

\section{FEATURES OF ORAL LEUKOPLAKIA}

Oral leukoplakia is the most common chronic keratotic lesion of the oral cavity and is potentially premalignant. ${ }^{1,2}$ It is a clinical diagnosis and is characterized predominantly by adherent white plaques of the oral mucosa. ${ }^{3}$

Oral leukoplakia has an estimated prevalence of $0.1 \%^{3}$ and is mostly found on the tongue of middle-aged and older men who smoke. ${ }^{1}$

Oral leukoplakia has various forms based on thickness, texture, color, and regularity, and each form has a different biologic behav-

doi:10.3949/ccjm.87a.19087

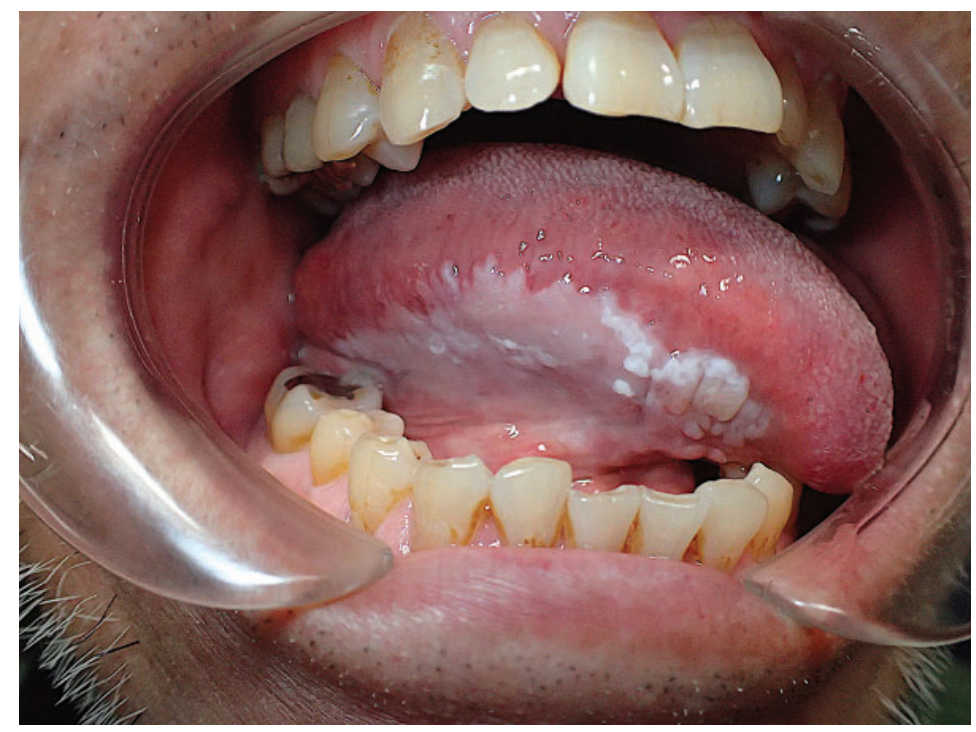

Figure 1. Homogeneous flat white plaque measuring 45 $\mathrm{mm} \times 20 \mathrm{~mm}$ and with slight corrugations was seen on the right lateral border of the tongue.

ior. $^{2}$ Tenderness and induration on palpation are indications of malignancy. ${ }^{1}$

Other conditions that can involve similar white plaques include oral lichen planus, candidiasis, lupus erythematosus, nicotinic stomatitis, graft-vs-host disease, white sponge nevus, frictional hyperkeratosis, and squamous cell carcinoma. ${ }^{1}$

\section{MANAGEMENT}

For the initial management of oral leukoplakia, incisional biopsy for histopathologic study is the gold standard. The histopathologic appearance of oral leukoplakia can range from hyperkeratosis to various degrees of epithelial dysplasia. ${ }^{3}$ The clinical diagnosis of leukoplakia should change to a pathohistologic diagnosis in the presence of in situ carcinoma, squamous cell carcinoma, or verrucous carcinoma. $^{3}$ 
Malignant transformation occurs in 2\% to $3 \%$ of cases. Features associated with a higher rate of malignant transformation are nonhomogeneous oral leukoplakia, presence of red lesions (erythroplakia), large lesion size (> $200 \mathrm{~mm}^{2}$ ), location on the tongue or floor of the mouth, age over 50 , female sex, no history of smoking, and the presence of severe dysplasia. ${ }^{4}$ Candida and human papillomavirus are infectious risk factors. ${ }^{1}$

The approach to management-ie, surgical resection, laser treatment, or careful monitoring - should be selected based on the histo- pathologic degree of dysplasia and on clinical features. ${ }^{2}$ Nonsurgical treatments have included administration of retinoids, vitamin C, beta carotene supplements, 5 -fluorouracil, and bleomycin. ${ }^{3}$

These treatments may be effective for resolution of the lesions; however, there is no evidence that they prevent malignant transformation. ${ }^{5}$

But regardless of the treatment, long-term observation and periodic biopsy are important to monitor for recurrence and for evidence of malignant transformation.

\section{REFERENCES}

1. Mangold AR, Torgerson RR, Rogers RS 3rd. Diseases of the tongue. Clin Dermatol 2016; 34(4):458-469.

doi:10.1016/j.clindermatol.2016.02.018

2. Awadallah M, Idle M, Patel K, Kademani D. Management update of potentially premalignant oral epithelial lesions. Oral Surg Oral Med Oral Pathol Oral Radiol 2018; 125(6):628-636. doi:10.1016/j.00oo.2018.03.010

3. van der Waal I. Oral leukoplakia: present views on diagnosis, management, communication with patients, and research. Curr Oral Health Rep 2019; 6(1):9-13. doi:10.1007/s40496-019-0204-8
4. Speight PM, Khurram SA, Kujan O. Oral potentially malignant disorders: risk of progression to malignancy. Oral Surg Oral Med Oral Pathol Oral Radiol 2018; 125(6):612-627. doi:10.1016/j.00oo.2017.12.011

5. Lodi G, Sardella A, Bez C, Demarosi F, Carrassi A. Interventions for treating oral leukoplakia. Cochrane Database Syst Rev. 2006;18(4):CD001829. doi: 10.1002/14651858.CD001829.pub3

Address: Keiichi Ohta, DDS, Department of Dentistry and Oral Surgery, Unit of Sensory and Locomotor Medicine, Division of Medicine, Faculty of Medical Sciences, University of Fukui, 23-3 Matsuoka, shimoaizuki, Eiheiji, Fukui, 910-1193, Japan; kohta@g.u-fukui.ac.jp

\section{F Cleveland Clinic \\ 32nd Annual Intensive Review of Internal Medicine} June $1-5,2020$ InterContinental Hotel and Bank of America Conference Center | Cleveland, $\mathrm{OH}$

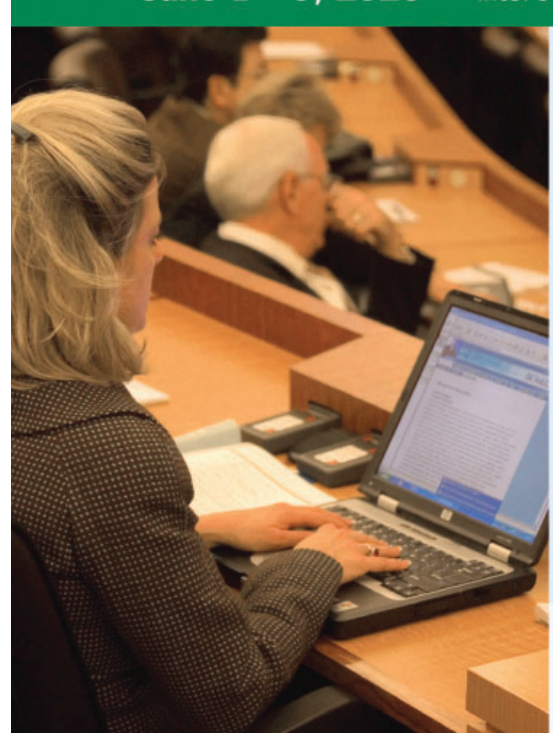

\section{HIGHLIGHTS}

Earn ABIM MOC Points

Mobile App for all Attendees!

Access presentations anytime, anywhere. Take notes, draw, or highlight

Audience Response System

Interactive case \& MCQ based talks with audience response system

Meet the Professor Lunches

Meet subspecialty speakers for one-on-one discussions

WiFi in Meeting Room

Cases from Cleveland Clinic Journal of Medicine

Access to useful clinical cases for boards and practice from Cleveland Clinic Journal of Medicine

This activity has been approved for AMA PRA Category 1 Credit $^{T M}$.

\section{Register Today! ccfcme.org/GolRIM}

